\title{
Fringe tracking performance monitoring: FINITO at VLTI
}

\author{
Mérand A. ${ }^{a}$; Patru F. ${ }^{a}$; Berger, J.-P. ${ }^{a}$; Percheron, I. ${ }^{b}$ and Poupar S. ${ }^{a}$ \\ ${ }^{a}$ European Southern Observatory, Alonso de Córdova 3017, Vitacura, Santiago, Chile; \\ ${ }^{b}$ European Southern Observatory, Karl-Schwarzschild-Straße 285748 Garching, Germany;
}

\begin{abstract}
Since April 2011, realtime fringe tracking data are recorded simultaneously with data from the VLTI/AMBER interferometric beam combiner. Not only this offers possibilities to post-process AMBER reduced data to obtain more accurate interferometric quantities, it also allows to estimate the performance of the fringe tracking a function of the conditions of seeing, coherence time, flux, etc. First we propose to define fringe tracking performance metrics in the AMBER context, in particular as a function of AMBER's integration time. The main idea is to determine the optimal exposure time for AMBER : short exposures are dominated by readout noise and fringes in long exposures are completely smeared out. Then we present this performance metrics correlated with Paranal local ASM (Ambient Site Monitor) measurements, such as seeing, coherence time or wind speed for example. Finally, we also present some preliminary results of attempts to model and predict fringe tracking performances, using Artificial Neural Networks.
\end{abstract}

Keywords: Optical Interferometry ; Fringe tracking ; Atmospheric Turbulence ;

\section{INTRODUCTION}

\subsection{Context}

The realtime data of the fringe tracking sensor (FINITO) and Optical Path Delay Controler (OPDC) have been recorded since April 1rst 2011 at the VLTI, $\frac{1}{1}$ whenever AMBER was used with FINITO with three telescopes. The motivation to offer such feature came after it has been demonstrated that the AMBER data reduction can benefit from this additional information. ${ }^{2,3}$ The real time fringe tracking data (hereafter RTFTD) come under the form of four additional binary tables in the AMBER FITS files containing records obtained thanks to the Reflective Memory Network recorder ${ }^{[4}$ The RTFTD recording frequency is of the order of the kilo-hertz, compared to AMBER data typical frame rate (between 0.1 and 10 hertz). This time resolution allows not only to reconstruct a posteriori what happened during the AMBER exposure,$\frac{3}{3}$ but also to derive the performances of the fringe tracking. In the context AMBER squared visibilities (the phase products are beyond the scope of this work), users are interested into basically two informations :

- Whether or not the fringe tracker loop was closed during a given AMBER frame, or in other words, whether or not the frame is useful for data reduction;

- What is the fringes contrast's loss in the science channel (i.e. AMBER) due to phase jitter residuals ;

Ideally, one wants the maximum number of useful frames over a given period of time, and with the highest contrast possible for each of these frames (smallest phase jitter). The reason the AMBER observations are truncated in short frames is that usually the longer exposure times, the higher the resulting phase jitter and the smaller the probability that the fringe tracker properly locked during the entire frame. Conversely, there is a limit of how short the exposure can be, since AMBER has a read-out noise limited detector (HAWAII infrared array).

Further author information: Send correspondence to amerand@eso.org, Telephone: +5624635311 


\subsection{Formalization}

We will start from the ideal expression of the signal-to-noise ratio of the AMBER single baseline visibility, as a function of $\mathrm{N}$ the total number of photo-electrons, $\mathrm{M}=T / \delta t$ the total number of frames ( $T$ is the total integration time, $\delta t$ the AMBER Detector Integration Time or DIT), $\sigma_{\text {RON }}$ the readout noise standard deviation (expressed in photo-electrons) :

$$
(S / N)(M) \sim \sqrt{M} \frac{N / M}{\sqrt{N / M+\sigma_{\mathrm{RON}}^{2}}}
$$

In this first expression, we ignore the fringe tracker all together, so this quantity is maximized for $\mathrm{M}=1$ : i.e. a single long exposure. In practice a single long exposure is not possible, since fringes eventually vanish due to the atmospheric turbulence, even if a fringe tracker is used. In the context of fringe tracking, we define $V_{\text {jitt. }}^{2}(\delta t)$ as the coherent loss due to the residual phase jitter, $L R(\delta t)$ the locking ratio (fraction of useful frames) and $n$ the photo-electrons flux (per unit of $\delta t$ ), so $N / M=n \delta t$ :

$$
(S / N)(\delta t) \sim \sqrt{L R(\delta t) T / \delta t} \frac{n \delta t \sqrt{V_{\mathrm{jitt} .}^{2}(\delta t)}}{\sqrt{n \delta t+\sigma_{\mathrm{RON}}^{2}}} \propto \sqrt{\delta t \frac{L R(\delta t) V_{\mathrm{jitt}}^{2}(\delta t)}{n \delta t+\sigma_{\mathrm{RON}}^{2}}}
$$

Note that we allowed ourselves to omit some proportional terms in this last expression, since we are only interested in maximizing this expression as a function of $\delta t$. From this expression, we can qualitatively analyse the two extreme cases : very short integration time (for the science channel) when we can expect the atmospheric turbulence to be frozen; and the long integration time (in the science channel) when we expect the science channel to be photon noise limited :

\begin{tabular}{|l|c|c|}
\hline $\begin{array}{l}\text { AMBER DIT }(\delta t) \\
\text { expected regime }\end{array}$ & $\begin{array}{c}\text { "short" } \\
\text { frozen turbulence }\end{array}$ & $\begin{array}{c}\text { "long" } \\
\text { photon noise limited }\end{array}$ \\
\hline$V_{\text {jitt. }}^{2}(\delta t)$ & $\sim 1$ & $<<1$ \\
$L R(\delta t)$ & $\sim 1$ & $<<1$ \\
$\frac{\delta t}{n \delta t+\sigma_{\mathrm{RON}}^{2}}$ & $<<1$ & $>>1$ \\
\hline $\mathrm{S} / \mathrm{N}$ & $<<1$ & $<<1$ \\
\hline
\end{tabular}

It seems clear from this qualitative analysis that neither short nor long DITs are a solution, and that an optimum lies in between. This optimum DIT depends on many parameters, some of which are related to the fringe tracker performance $\left(V_{\mathrm{jitt}}\right.$ and $\left.L R\right)$, the science detector $\left(\sigma_{\mathrm{RON}}\right)$ and the target brightness $(n)$.

\section{RESULTS}

\subsection{Data Analysis}

From the RTFTD, it is easy to derive an estimate of the two quantities of interest $: L R$ and $V_{\text {jitt. }}^{2}$ The locking ratio is readily computable by a sliding minimum operator on the control loop state machine : the OPDC state machine is state '7' when fringe tracking, '5' idle (fringes deemed too low to close the loop), '4' when it is only tracking the group delay and so on. We apply a sliding windowed minimum operator with a given time window in order to compute the average locking ratio other a given data file (usually covering one minute). Conversely, we compute $V_{\text {jitt. }}^{2}$ using the classical coherent loss formula $5 V_{\text {jitt. }}^{2}=\exp -\left(2 \pi \sigma_{\mathrm{OPD}} / \lambda\right)^{2}$ where $\sigma_{\mathrm{OPD}}^{2} / \lambda^{2}$ is the phase variance of the optical path delay (piston) residual and $\lambda$ the wavelength of the scientific instrument (AMBER, which mostly operate in the $\mathrm{K}$ band, $\lambda=2.2 \mu \mathrm{m}$ ). It is remarkable to note that this quantity is actually a generalized Strehl Ratio but instead of the classical definition involving the standard deviation of the wavefront of an optical instrument, it involves the temporal standard deviation of the phase variation. Because this temporal standard deviation is what is actually measured in the RTFTD, we will sometimes use $\sigma_{\mathrm{OPD}}$, computed using a sliding variance operator for a given time window, instead of the interferometric Strehl $V_{\text {jitt. }}^{2}$ which depends on the wavelength of the scientific channel. Figure 1 explains visually how we analysed the data, based on a small time sequence.

On Fig. 2, we show the overview of different files' analysis, as a function of the time window : as expected, the interferometric Strehl and locking ratio decrease as the time window increases. The two have slightly different behaviors : 


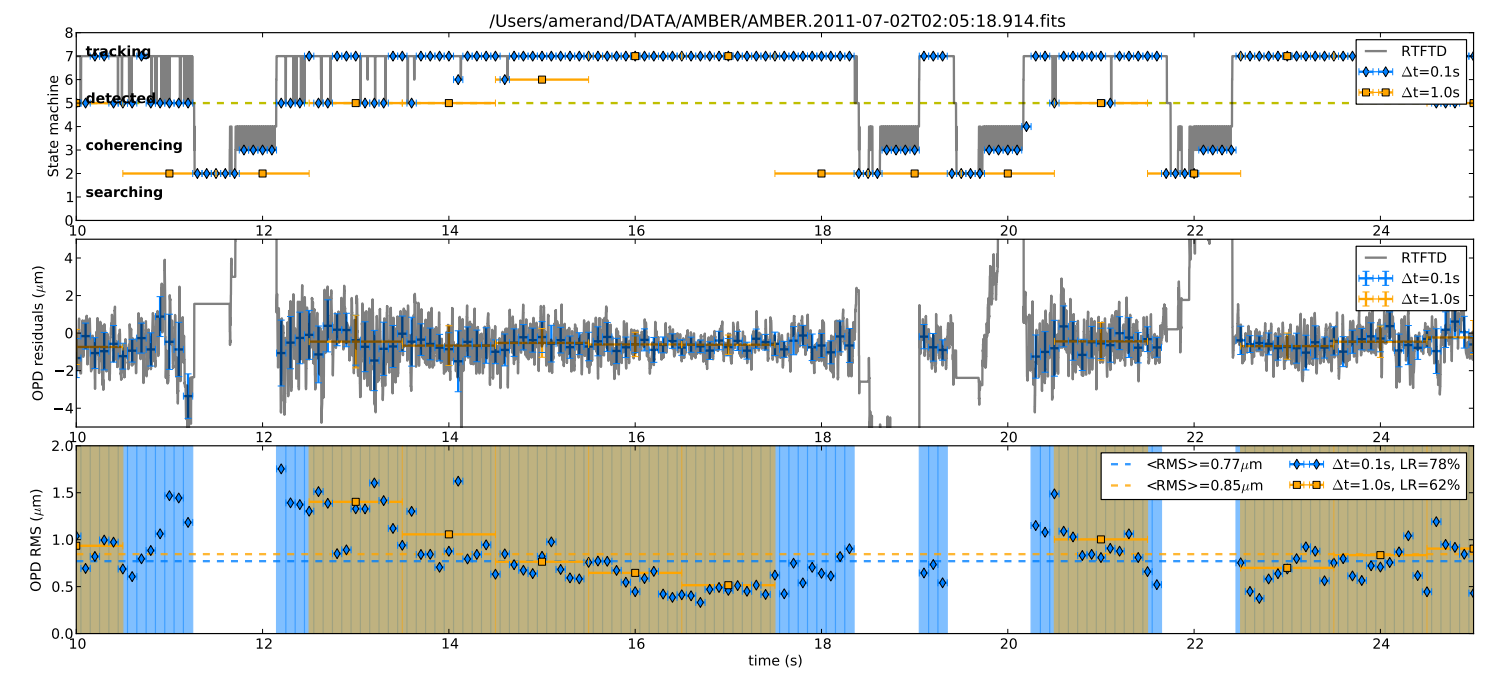

FIGURE 1. Small RTFTD time sequence explaining the data analysis based on sliding operators with variable time windows. For clarity, only 0.1 and 1.0s time windows are shown. The upper panel shows the fringe tracker state machine and its different levels (tracking, fringes detected, coherencing - i.e. group delay tracking only — and searching) and the sliding operator used to determine the minimum state during an exposure. Its value must be $\geq 5$ for the frame to be considered useful. The middle pannel show the OPD tracked by fringe tracker. The different point show the sliding average and standard deviations. Points are ommited when the loop is open (state $\leq 4$ ). The lower panel show the OPD RMS for the useful frames. Note that the shorter time window has more useful frames and lower RMS. See the electronic version for a color version of this figure.

the interferometric Strehl is decreasing up to time windows of the order of $0.1 \mathrm{~s}$ and reaches then a relatively shallow slope. On the other hand, the locking ratio decreases linearly with time (though it is not so clear on the semi-log plot), ultimately reaching 0 . Poor conditions are characterized by low interferometric Strehl and low lock rate; typical conditions are characterized by high interferometric Strehl but dropping locking rate for DITs of 1s or larger; good conditions have high interferometric Strehl and lock rate dropping for DITs longer than 10s or so. Applying the SNR formula, we can compute the optimum AMBER DIT (Fig. 2). The behavior of the SNR as a function of the time window has two regimes : first it increases as expected as the read out noise gets less and less important and the fringe tracking performances are relatively stable, then it drops rapidly as the lock ratio drops.
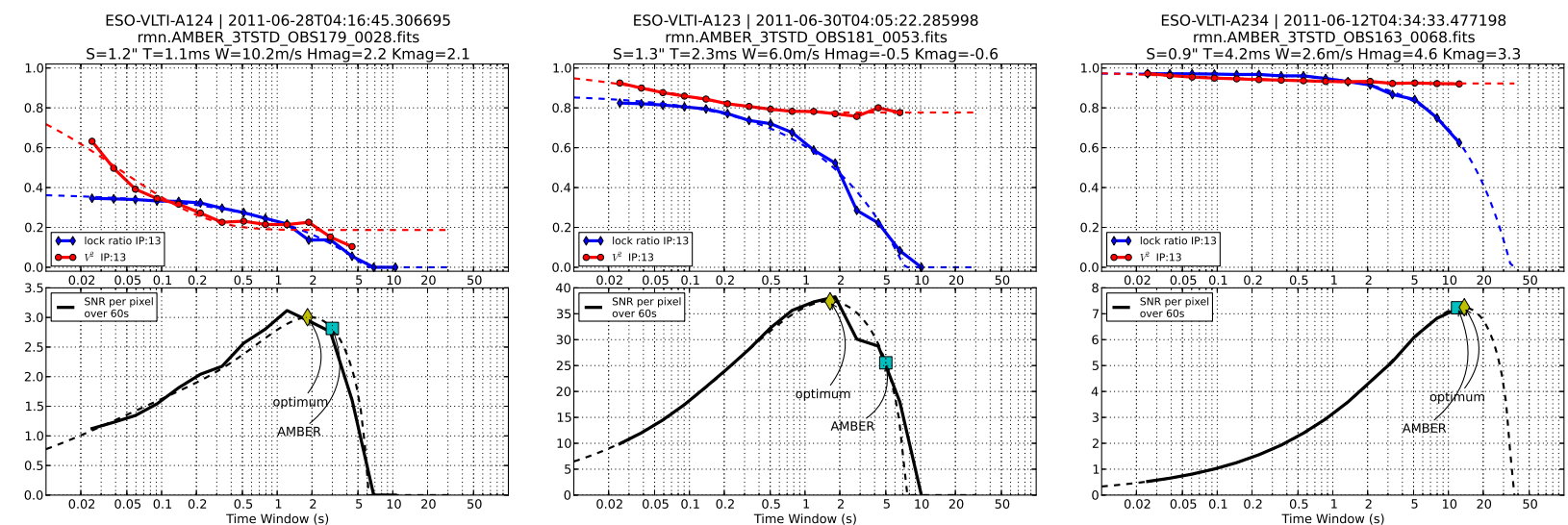

FIGURE 2. Single AMBER/FINITO file Analysis for bad (left), typical (center) and good (right) atmospheric conditions. For each panel, the upper subpanel shows the locking ratio (blue) and visibility loss (red) as a function of time window (i.e. the integration in AMBER). The lower subpanel shows the expected SNR per pixel reachable in 60 seconds in AMBER. The optimum DIT and the actual AMBER DIT used in the data are shown. The dash lines are a simple analytical fit to the data, in order to unambiguously determine the optimum DIT (monotonous derivative). See the electronic version for a color version of this figure. 


\subsection{Global Analysis : The case of the Auxiliary Telescopes (AT)}

\subsubsection{Wind and coherence time}

We present in this section a global analysis of one year of FINITO/AMBER RTFTD for observations performed with the $1.8 \mathrm{~m}$ diameter Auxiliary Telescopes (ATs), limited to the analysis of calibrator stars which have by definition high and predictable fringes' visibilities. The fringe tracker performances are expected to vary with the atmospheric parameters. In order to assess this quantitatively, we will use the Ambient Conditions Database ${ }^{*}$ that records parameters such as wind speed and directions, as well as the seeing and coherence time from Paranal's DIMM (differential Image Motion Monitor). The DIMM values are computed for visible wavelength $(550 \mathrm{~nm})$ and for 0 degree zenithal distance. These values are readily available in the header of the FINITO/AMBER FITS files.
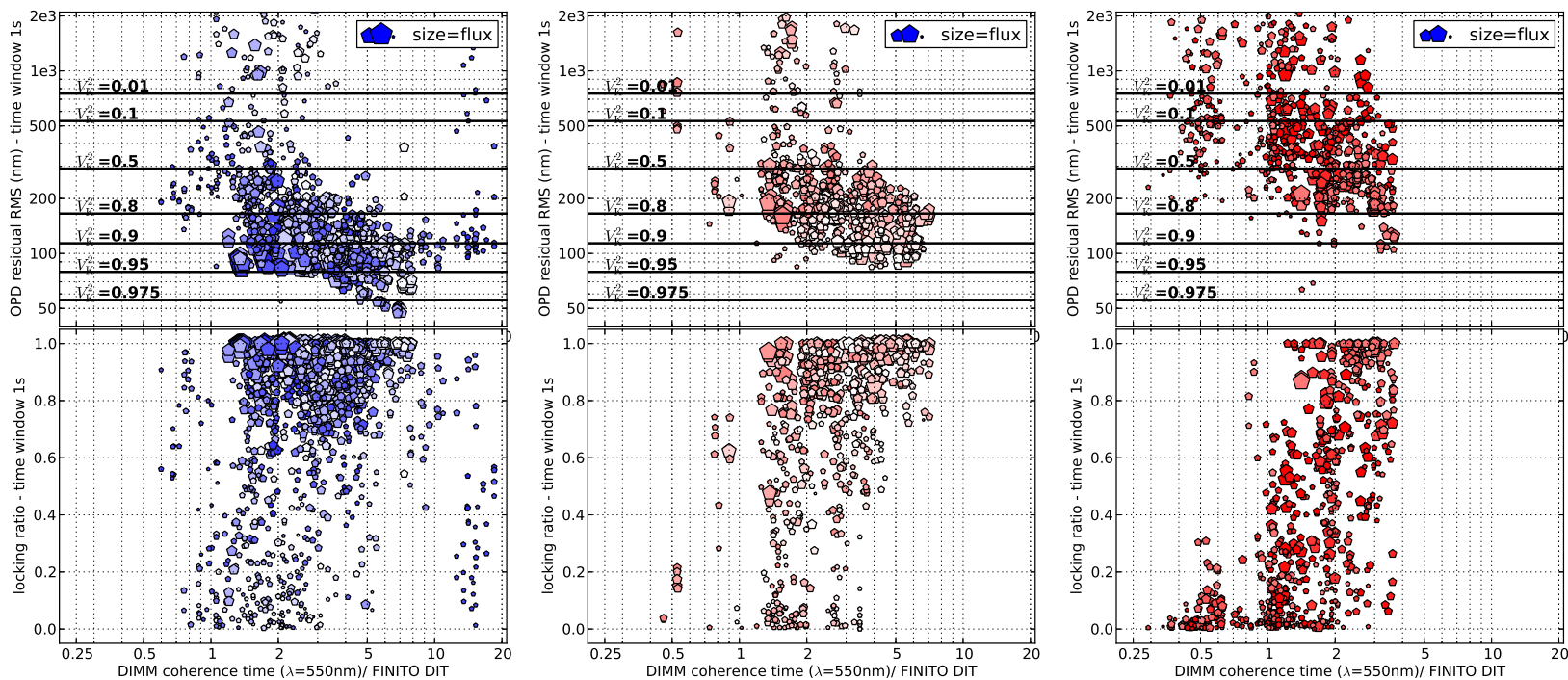

Figure 3. 1 year of Auxiliary Telescopes (AT) data, only for calibrators (predictable visibility). Fringe tracking performances : OPD rms and $V_{K}^{2}$ the interferometric Strehl in $\mathrm{K}$ band (upper panels) and locking ratio (lower panels) for 1s time window. The performances parameters are plotted as a function of DIMM coherence time divided by the exposure time in FINITO (horizontal axis) and flux measured in FINITO (size of the dots). For clarity we distinguished low wind ( left, WS $\leq 6 \mathrm{~m} / \mathrm{s}$ ), medium wind (middle, $6 \leq \mathrm{WS} \leq 10 \mathrm{~m} / \mathrm{s}$ ) and high wind (right, WS $\geq 10 \mathrm{~m} / \mathrm{s})$. Wind speeds is color coded from blue $(0 \mathrm{~m} / \mathrm{s})$, white $(6 \mathrm{~m} / \mathrm{s})$ to red $(12 \mathrm{~m} / \mathrm{s}$ and more). See the electronic version for a color version of this figure.

In Fig. 3. we plotted the performance as a function of the DIMM coherence time at wavelength 550nm (in s), multiplied the FINITO frame rate (in Hz, typically $1 \mathrm{kHz}$ ). It is to be noted that the DIMM coherence time is based on the DIMM seeing and a combination of wind prediction in the upper atmosphere and wind measured locally (at altitudes of $10 \mathrm{~m}$ and $30 \mathrm{~m}$ ). It is thus indicative of the atmosphere turbulence coherence time rather than a true measurement. This figure shows that the dependency is mostly shallow in coherence time, except for the low coherence time (high turbulence) when fringe tracking performances show dramatic non linearity. It is also interesting to notice the strong dependency with wind speed. Here are the typical performances, for $1 \mathrm{~s}$ time window, within operational limits (DIMM coherence time of the order of $2 \mathrm{~ms}$ of more) :

\begin{tabular}{|l|c|c|c|}
\hline performance & $\begin{array}{c}\text { low wind } \\
1 \text { s time window }\end{array}$ & $\begin{array}{c}\text { medium wind } \\
\leq 6 \mathrm{~m} / \mathrm{s}\end{array}$ & $\begin{array}{c}\text { high wind } \\
\geq 6 \mathrm{~m} / \mathrm{s} \text { and } \leq 10 \mathrm{~m} / \mathrm{s}\end{array}$ \\
\hline OPD RMS & $50-150 \mathrm{~nm}$ & $100-250 \mathrm{~nm}$ & $\geq 300 \mathrm{~nm}$ \\
interf. Strehl K & $0.8-0.95$ & $0.6-0.85$ & $\leq 0.5$ \\
lock ratio & $\sim 0.8$ & $\sim 0.75$ & anything \\
\hline
\end{tabular}

It is not clear yet if the dependency with the wind speed results from the fact that the coherence time formula used is biased with local wind speed, or if FINITO is badly affected by ATs optical tube vibrations, since these telescopes have an open concept : the hemispherical dome is retracted during observations. The answer is probably a combination of both.

*. http://archive.eso.org/asm/ambient-server 


\subsubsection{Limiting magnitude}

Additionally, one can note that as expected, the performances depend on the flux received in FINITO (the size of each dot on the plots) : For low wind conditions, most low interferometric Strehl ratio and/or lock ratio are observed for low flux, which is expected in a service mode where most challenging observations are executed in the best conditions. Based of the available data, the limiting magnitude is estimated to be of the order of correlated $\mathrm{H}$ magnitude of 5.5, which is what is guarantied currently in service mode under good atmospheric conditions.

Comparing this number to the originally expected ${ }^{\sqrt{6}}$ limiting magnitude of $\mathrm{H}=10$ may rise questions. Actually, most of the loss were known early on : a worst that expected read out noise $\left(40 \mathrm{e}^{-}\right.$instead of $\left.5 \mathrm{e}^{-6}\right)$; worst than expected transmission $\left(0.25\right.$ instead of $\left.0.6^{6}\right)$ and optimistic expectation regarding the operational frame rate $\left(1000 \mathrm{~Hz}\right.$ instead of $\left.100 \mathrm{~Hz} z^{6}\right)$. From the present data, we computed using the measured transmission of the VLTI, that the turbulent coupling in the single mode fibers leads to an additional transmission term of the order of 0.2 or 0.3 , even in the best conditions. All this taken into account leads to a revised expected limiting magnitude of Hmag 5.0 on the ATs, which is what we see in practice.

\subsubsection{Optimum DIT and limiting magnitude gain}

We recall that we call optimum DIT the exposure time that will best mitigate the loss of fringe tracking performances for long integration times, and the necessity to increase exposure time in the science channel in order to reach the photon noise limited regime, as opposed to the read-out noise limited regime. For the ATs, we see that the typical optimum DIT for $\mathrm{K}$ band science using FINITO lies between 1s and 10s, depending on the conditions. The typical DIT we use in AMBER without FINITO is of the order of 100ms. That means FINITO effectively provides a gain of 4 magnitudes.

This 4 magnitudes gain does not necessarily translate into a gain in limiting magnitude for AMBER. For instance, it does not help in the case of low spectral resolution (LR) in AMBER observations, since in this mode AMBER is roughly equally sensitive as FINITO. In other words, faint targets for AMBER/LR that would benefit from longer exposure times and stabilized OPD are not reachable by FINITO. However there is a clear gain for higher spectral resolution modes, $\mathrm{R} \sim 1500$ and $\mathrm{R} \sim 15000$ for MR and HR modes, which are severely limited in term of sensitivity in the free atmosphere regime.

\subsection{Global analysis : the case of the Unit Telescopes (UTs)}

The case of the UTs is different : what the fringe tracking needs to fight in that case is not only the atmospheric turbulence but also the intrinsic vibrations of the telescopes themselves: ${ }^{7}$ It turns out (see Fig. 4 th the resulting fringe tracking performances on the UTs is dramatically worse than encountered for the ATS (Fig. 3). In particular, the interferometric Strehl is very low, always less than 0.5 , with a typical value of 0.1 .

Because data on all the baselines (i.e. UT pairs), it is possible to derive the contribution of each telescope. We derived the following OPD RMS :

\begin{tabular}{|c|cccc|}
\hline & UT1 & UT2 & UT3 & UT4 \\
\hline UT1 & $\sim \mathbf{3 7 7 n m}$ & $411 \mathrm{~nm}$ & $445 \mathrm{~nm}$ & $738 \mathrm{~nm}$ \\
UT2 & - & $\sim \mathbf{2 1 5 n m}$ & $402 \mathrm{~nm}$ & $548 \mathrm{~nm}$ \\
UT3 & - & - & $\sim \mathbf{2 8 7 n m}$ & $580 \mathrm{~nm}$ \\
UT4 & - & - & - & $\sim \mathbf{5 4 2} \mathrm{nm}$ \\
\hline
\end{tabular}

Where the non diagonal terms are the measured residuals for the pair of telescopes, and the diagonal terms are the deduced single telescope contributions. This is to be compared to the contributions of the 3 first mirrors (M1, M2, M3) using accelerometers ${ }^{7]}: 190,170,240$ and 300nm for UT1, UT2, UT3 and UT4 respectively. The additional RMS we detect is due to a combination of atmospheric effects and undetected vibrations by the vibration tracking system which only account for part of the optical path. 

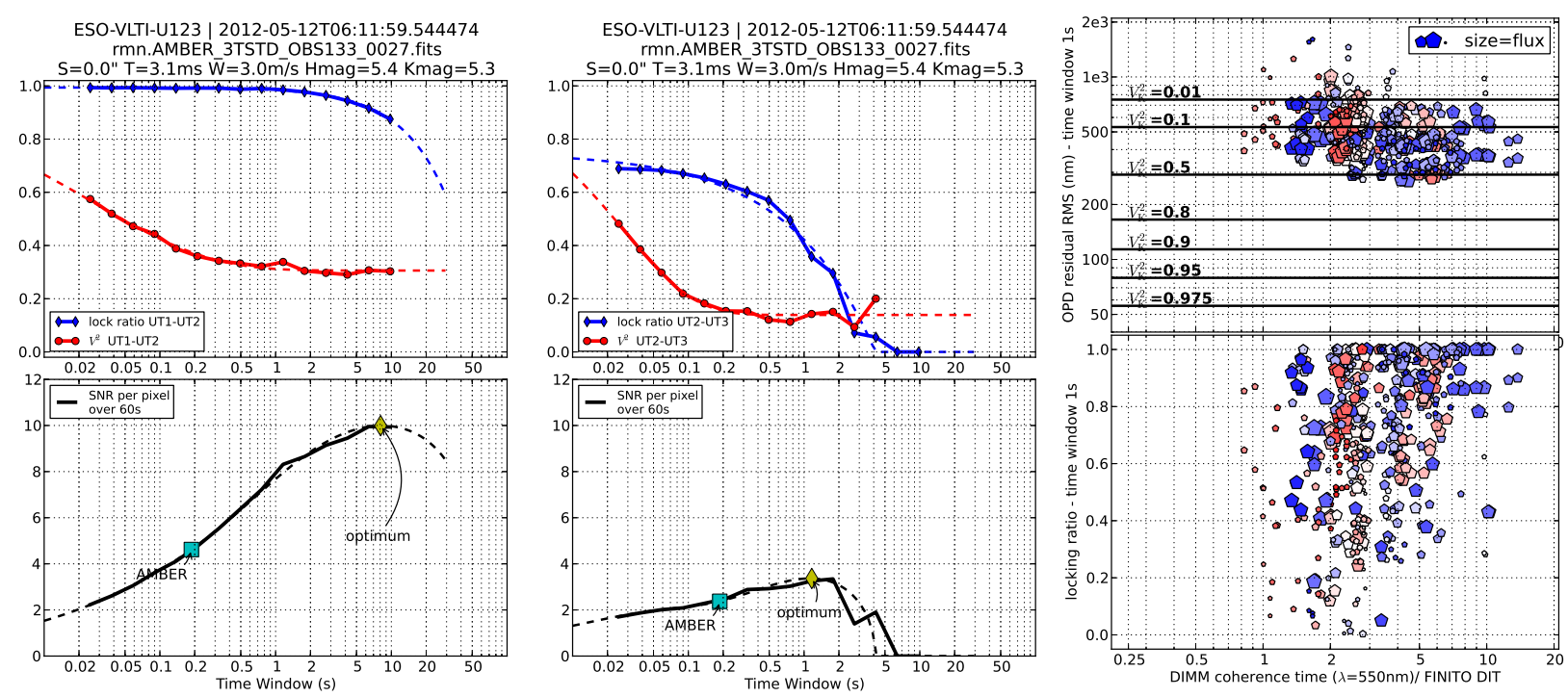

FiguRE 4. Left and middle panels : same figure as Fig. 2 but for the UTs under good conditions. The same observation is shown from the perspective of UT1-UT2 (left) and UT2-UT3 (middle) showing the difference in performances attributable to the different level of vibrations between UT1, UT2 and UT3, this later having the worst vibration level. On the right, plot similar to Fig. 3 but for the UTs, the only difference is that the flux scaling is not comparable. Colors gradient correspond to wind speed : blue is no wind, white is medium wind $(6 \mathrm{~m} / \mathrm{s})$ and red if high wind $(12 \mathrm{~m} / \mathrm{s})$. See the electronic version for a color version of this figure.

\section{DISCUSSION}

\subsection{Impact on operations}

The analysis we have been presenting has not had any impact on operations yet : service mode is based on a queue containing Observations Blocks prepared in advance. The DIT of AMBER is set according to a table depending on the spectral resolution, magnitude of the object and requested seeing conditions (as a proxy to atmospheric conditions). The DIT abacus is currently based on experience rather than the type of analysis we presented. We can now retrospectively compare our current strategy to choose the DIT with a reasoned one we presented.
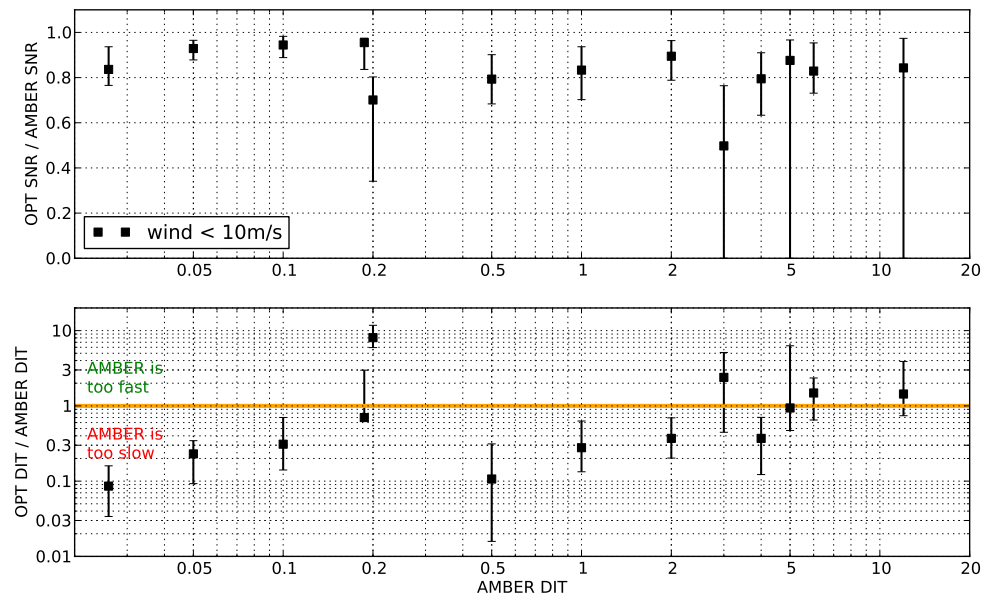

FIGURE 5. Comparison between computed optimum DIT and actual DIT used in the file (lower panel), and the resulting loss in SNR (upper pannel) as a function of AMBER DIT. These data are for low wind conditions $(\leq 10 \mathrm{~m} / \mathrm{s})$. Each point is the median, with the error bar being the first and third quartile. See the electronic version for a color version of this figure.

For each file, we can computed the optimum DIT and the associated maximum expected SNR, compared to the AMBER DIT and expected AMBER SNR. As seen on Fig. 5, comparing the used DIT and the optimum one, in low wind conditions, 
the loss in SNR is usually small : of the order of 10 to $20 \%$ only. In general, we run AMBER with a DIT that is longer that it should be. This is actually a bit dangerous, because of the behavior of the locking ratio that drops as a function of exposure time : it is safer to actually use a DIT which is too small rather than the opposite, because running long exposures might lead to no frame being taken with the loop continuously closed.

\subsection{UTs compared to ATs}

VLTI is has an interesting concept regarding performance investigation, in the sense that it is operated with two different types of telescopes : UTs and ATs. ATs are small (1.8m in diameter) and entirely dedicated to interferometry. UTs, on the other hand, are large ( $8.2 \mathrm{~m}$ in diameter) and more complex due to their multi-purpose nature that includes 3 instruments (2 Nasmith and 1 Cassegrain focii). The gain in sensitivity between UTs and ATs due to the M1 size should be of the order of 3.3 magnitudes, helped by the fact that UTs are equipped with Adaptive Optics. In practice, if we compare the actual fringe tracking observations made with the UTs to the ones with the ATs, the gain in limiting magnitude seems to be more of the order of 1.5 magnitude. Clearly, vibrations are a strong limitation to the full interferometric potentiel of the UTs.

\subsection{Perspectives : predicting performances}

A last aspect we have been investigating is the possibility to predict the fringe tracking performances as a function of environmental parameters (DIMM seeing, DIMM coherence time, local wind speed) and target characteristics (H magnitude, expected visibility). Fringe tracking performances are usually very non-linear with these parameters, in the sense that a light degradation of the conditions can dramatically reduce the performances. One obvious application of predicting the performances is estimate before hand the optimum DIT in the case of conditions at the limit of the operable regime. If the conditions are good and the target is bright, one can qualitatively predict that fringe tracking will work, conversely if conditions are bad and/or the target is too faint. Modeling the intermediate regime, due to its non linearities (chaotic atmosphere, complex state machines and control loops, etc.), proves to be difficult.

We have investigated a relatively simple ad-hoc approach using Artificial Neural Networks (ANN) to try to predict high level performances parameters such as the locking ratio and RMS residuals. We tried to use our data set to train an ANN using the DIMM seeing, DIMM coherence time, local wind speed and target $\mathrm{H}$ correlated magnitude as the input of the model; and locking ratio and OPD RMS over 1s as the output.

We have just started to work on this aspect and the results of the training (using a back propagation algorithm) is shown of figure 6 where we compare the training set and the prediction, after the ANN has been trained. The results are encouraging but we will not draw yet any conclusion yet.
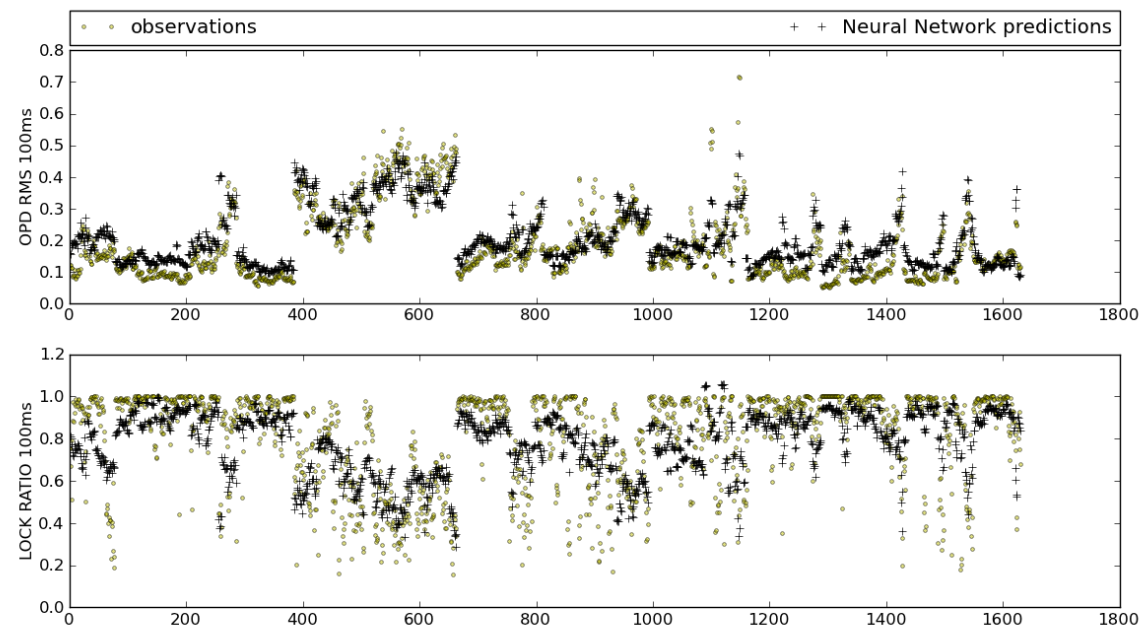

FIGURE 6. Artificial Neural Network prediction of the fringe tracking performances : upper panel shows the OPD rms over 100ms (in microns) and the lower panel shows the locking ratio over 100ms. The horizontal axis is the record number in the learning sample. See the electronic version for a color version of this figure. 


\section{CONCLUSIONS}

We have presented a quantitative analysis of one year of real time fringe tracking data (RTFTD) of FINITO/AMBER at VLTI. We have analyzed separately observations obtained with the Auxiliary Telescopes (ATs) and the Unit Telescopes (UTs) because of the different limitations that affect these two types of telescopes. We have shown that the FINITO reaches good performances : path delay residuals RMS of less than 150nm, corresponding to an interferometric Strehl (atmospheric residual fringe contrast) of $80 \%$ or higher in $\mathrm{K}$ band, for typical atmospheric conditions. These performances are obtained for equivalent integration time (for the science channel) of one to ten seconds typically. The main limitations in term of performance of the FINITO fringe tracker seem to be :

- The inability to maintain the control loops for periods of time longer that 10 seconds or so. This is mostly due to the strategy to estimate and track the group delay. It is to be noted that the other fringe tracker at VLTI, the PRIMA's FSU, do not suffer from this problems, thanks to a different design.

- The sensitivity which suffers from the usual culprits : lower than expected instrumental transmission and higher than expected read-out noise of the infrared detector, which would allow a gain of 3 magnitudes or more if they were within specifications.

- The degradation of the phase residuals (hence a decreased interferometric Strehl ratio) when the wind blows on the ATs at speeds above $10 \mathrm{~m} / \mathrm{s}$;

- The detrimental effect of the UTs' intrinsic vibrations which put them at the level of the ATs in the worse atmospheric conditions (low coherence time and wind speed above $12 \mathrm{~m} / \mathrm{s}$ ) in term of fringe tracking performances.

We have introduced the notion of optimum DIT (for the science channel, here AMBER) for which the signal to noise ratio of combined frames over a given period of time is maximized. It turns out we currently operate AMBER close to its optimum DIT when FINITO is used, even if the analysis presented here is not yet performed in real time nor used to adjust AMBER's DIT.

Future developments of this work may include : monitoring of the overall performances of VLTI; advanced data reduction for $\mathrm{AMBER}^{[3}$; real time RTFTD analysis and/or performance predictions to improve operations.

\section{REFERENCES}

[1] Haguenauer, P., Alonso, J., and Bourget, P. e. a., "The very large telescope Interferometer : 2010 edition," in [Society of Photo-Optical Instrumentation Engineers (SPIE) Conference Series], Society of Photo-Optical Instrumentation Engineers (SPIE) Conference Series 7734 (July 2010).

[2] Le Bouquin, J.-B., Abuter, R., and Haguenauer, P. e. a., "Post-processing the VLTI fringe-tracking data : first measurements of stars," AEA 493, 747-752 (Jan. 2009).

[3] Mérand, A., Stefl, S., and Bourget, P. e. a., "Perspectives for the AMBER Beam Combiner," in [Society of PhotoOptical Instrumentation Engineers (SPIE) Conference Series], Society of Photo-Optical Instrumentation Engineers (SPIE) Conference Series 7734 (July 2010).

[4] Abuter, R., Popovic, D., and Pozna, E. e. a., "The VLTI real-time reflective memory data streaming and recording system," in [Society of Photo-Optical Instrumentation Engineers (SPIE) Conference Series], Society of Photo-Optical Instrumentation Engineers (SPIE) Conference Series 7013 (July 2008).

[5] Colavita, M. M., "Fringe Visibility Estimators for the Palomar Testbed Interferometer," PASP 111, 111-117 (Jan. 1999).

[6] Gai, M., Menardi, S., and Cesare, e. a., "The VLTI fringe sensors : FINITO and PRIMA FSU," in [Society of PhotoOptical Instrumentation Engineers (SPIE) Conference Series], Traub, W. A., ed., Society of Photo-Optical Instrumentation Engineers (SPIE) Conference Series 5491, 528 (Oct. 2004).

[7] Poupar, S., Haguenauer, P., and Merand, A. e. a., "Status of the VLTI-UT performances wrt vibrations," in [Society of Photo-Optical Instrumentation Engineers (SPIE) Conference Series], Society of Photo-Optical Instrumentation Engineers (SPIE) Conference Series 7734 (July 2010). 